\title{
MODELAGEM MATEMÁTICA DA SECAGEM DE FRUTOS DE CRAMBE EM CAMADA DELGADA
}

\author{
MATHEMATICAL MODELING OF CRAMBE FRUITS IN THIN LAYER DRYING
}

\author{
Lílian Moreira COSTA ${ }^{1}$; Osvaldo RESENDE ${ }^{1}$; Douglas Nascimento GONÇALVES ${ }^{1}$; \\ Daniel Emanuel Cabral de OLIVEIRA ${ }^{1}$ \\ 1. Instituto Federal Goiano, Rio Verde, GO, Brasil. 1mctpg@yahoo.com.br
}

\begin{abstract}
RESUMO: Objetivou-se no presente trabalho ajustar diversos modelos matemáticos ao processo de secagem dos frutos de Crambe abyssinica, em diversas condições de ar, bem como determinar o coeficiente de difusão efetivo e obter a energia de ativação. O trabalho foi realizado no Laboratório de Pós-colheita de Produtos Vegetais do Instituto Federal de Educação, Ciência e Tecnologia Goiano - Câmpus Rio Verde, Goiás, Brasil (IF Goiano - Câmpus Rio Verde). A colheita do crambe (Crambe abyssinica Hochst) da cultivar FMS Brilhante foi realizada manualmente, com o teor de

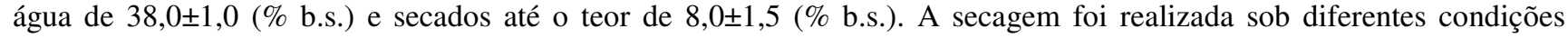
controladas de temperatura $35,45,60,75$ e $90{ }^{\circ} \mathrm{C}$ e umidades relativas de 20,$9 ; 8,7 ; 6,8 ; 4,8$ e $2,3 \%$, respectivamente. Dentre os modelos analisados, Page foi o melhor que se ajustou aos dados nas diferentes condições do ar de secagem. $\mathrm{O}$ coeficiente de difusão efetivo aumentou com a elevação da temperatura apresentando valores de $2,84 \times 10^{-11} ; 3,22 \times 10^{-11}$; $5,00 \times 10^{-11} ; 7,27 \times 10^{-11}$ e $9,14 \times 10^{-11} \mathrm{~m}^{2} \mathrm{~s}^{-1}$, para as temperaturas de $35,45,60,75$ e $90{ }^{\circ} \mathrm{C}$, respectivamente, durante a secagem dos frutos de crambe. A relação entre o coeficiente de difusão efetivo e a temperatura de secagem pode ser descrita pela equação de Arrhenius, que apresenta uma energia de ativação para a difusão líquida na secagem de 20,998 kJ $\mathrm{mol}^{-1}$.
\end{abstract}

PALAVRAS-CHAVE: Crambe abyssinica. Cinética de secagem. Coeficiente de difusão. Energia de ativação.

\section{INTRODUÇÃO}

A cultura do Crambe (Crambe abyssinica Hochst) surge com grande potencial para a produção de matéria-prima para fins industriais, e também como oleaginosa a ser utilizada como matéria-prima para produção de biodiesel (MÔLLER, 2011). O Crambe quando é prensado com o uso de solventes (o hexano, por exemplo) apresenta um teor de óleo de 35\% (FUNDAÇÃO MS, 2010) este óleo produzido não é comestível e tem sua utilização destinada a indústria oleoquímica e mais recentemente à produção de biodiesel. A conversão de óleo de crambe em biodiesel mostra-se viável, apresentando boa porcentagem de ácido graxo convertido em éster metílico (MACHADO, 2007).

Durante os últimos anos a cultura foi testada no Mato Grosso do Sul, com sucesso para a adubação verde e produção de grãos, para uso como matéria prima na produção de biodiesel, destacando que as pesquisas realizadas pela Fundação MS, de Maracaju apontaram para uma produção entre 1.000 e 1.500 quilos por hectare, conforme Baez (2011). A utilização do crambe para fins industriais vem aumentando a cada ano, no entanto para que se atenda a demanda é necessário o desenvolvimento de técnicas de manejo que possam vir a agregar uma maior produtividade (VECHIATTO; FERNANDES, 2011).
A secagem dos produtos agrícolas é o processo mais utilizado para assegurar sua qualidade e estabilidade considerando que a diminuição da quantidade de água do material reduz a atividade biológica e as mudanças químicas e físicas que ocorrem durante o armazenamento (RESENDE et al., 2008).

No desenvolvimento e aperfeiçoamento de equipamentos utilizados para a secagem de grãos, é de fundamental importância à simulação e a obtenção de informações teóricas a respeito do comportamento de cada produto durante a remoção de água. Para a simulação, cujo princípio se fundamenta na secagem de sucessivas camadas delgadas do produto, utiliza-se um modelo matemático que representa, satisfatoriamente, sua perda de água durante o período de secagem (GINER; MASCHERONI, 2002).

As curvas de secagem em camada delgada variam com a espécie, variedade, condições ambientais, métodos de preparo pós-colheita, entre outros fatores. Neste sentido, diversos modelos matemáticos têm sido utilizados para descrever o processo de secagem de produtos agrícolas (RESENDE et al., 2009; RESENDE et al., 2010).

A representação matemática do processo de secagem de diversos produtos agrícolas vem, já há algum tempo, sendo estudada e utilizada na tentativa de predizer os fenômenos que ocorrem durante este processo. Inúmeros modelos matemáticos têm sido 
estudados com o objetivo de projetar, controlar e otimizar processos secagem (ROCHA et al., 2005). A secagem consiste na remoção de grande parte da água contida no produto até determinado nível, originando condições desfavoráveis à continuidade de suas atividades metabólicas e ao desenvolvimento de microrganismos (MARTINAZZO et al., 2007).

Segundo Vilela e Artur (2008) as informações contidas nas curvas de secagem são de fundamental importância para o desenvolvimento de processos e para o dimensionamento de equipamentos; com elas, pode-se estimar o tempo de secagem de certa quantidade de produtos e, com o tempo necessário para a produção, estima-se o gasto energético que refletirá no custo de processamento e, por sua vez, influenciará no preço final do produto. No dimensionamento de equipamentos se pode determinar as condições de operação para secagem e, com isto, a seleção de trocadores de calor, ventiladores e outros.

Assim, objetivou-se, no presente trabalho, ajustar modelos matemáticos ao processo de secagem dos frutos de crambe, em diversas condições de ar, definindo o melhor modelo, bem como determinar o coeficiente de difusão efetivo e obter a energia de ativação para o processo.

\section{MATERIAL E MÉTODOS}

Este trabalho foi realizado no Laboratório de Pós-colheita de Produtos Vegetais do Instituto Federal de Educação, Ciência e Tecnologia Goiano - Campus Rio Verde, Goiás, Brasil (IF Goiano Câmpus Rio Verde). A colheita do crambe (Crambe abyssinica Hochst) da cultivar FMS Brilhante foi realizada manualmente, com o teor de água de $38,0 \pm 1$ (\% b.s.).

A secagem foi realizada em estufa, com ventilação forçada, nas seguintes condições de ar: temperaturas de secagem de $35,45,60,75$ e $90{ }^{\circ} \mathrm{C}$ e umidades relativas de 20,$9 ; 8,7 ; 6,8 ; 4,8$ e $2,3 \%$, respectivamente.

A temperatura e a umidade relativa do ar de secagem foram monitoradas por meio de um psicrômetro instalado no interior do secador. Durante o processo de secagem as bandejas com $400 \mathrm{~g}$ de amostras e $2 \mathrm{~cm}$ de espessura, com quatro repetições por temperatura, foram pesadas periodicamente até o teor de água de 8,0 b.s.).

Os teores de água do produto foram determinados por gravimetria utilizando-se estufa a $105 \pm 1{ }^{\circ} \mathrm{C}$, durante $24 \mathrm{~h}$ (BRASIL, 2009).

Para a determinação das curvas de secagem e ajustes dos modelos, o teor de água de equilíbrio para cada condição estudada foi obtido por meio do modelo de Copace, que melhor representa a higroscopicidade dos frutos de crambe (COSTA et al., 2012a).

$\mathrm{Xe}^{*}=\exp \left[-3,6064-(0,0335 \cdot \mathrm{T})+\left(1,5894 \cdot \mathrm{a}_{\mathrm{w}}\right)\right]$

em que: $X_{\mathrm{e}}^{*}$ : teor de água de equilíbrio, (\% b.s.); T: temperatura de secagem, ${ }^{\circ} \mathrm{C} ; \mathrm{A}_{\mathrm{w}}$ : atividade de água, decimal.

As razões de teor de água do produto foram determinadas pela expressão:

$$
\mathrm{RX}=\frac{\mathrm{X}^{*}-\mathrm{X}_{\mathrm{e}}^{*}}{\mathrm{X}_{\mathrm{i}}^{*}-\mathrm{X}_{\mathrm{e}}^{*}}
$$

em que: RX: razão de teor de água, adimensional; $X^{*}$ : teor de água do produto (\% b.s.); ${ }^{*}$ : teor de água inicial do produto (\% b.s.).

Aos dados experimentais da secagem dos frutos de crambe foram ajustados modelos matemáticos utilizados por diferentes pesquisadores para representação da secagem de produtos agrícolas conforme descritos na Tabela 1.

Tabela 1. Modelos matemáticos utilizados para predizer a secagem de produtos agrícolas.

\begin{tabular}{ll}
\hline Designação do modelo & Modelo \\
\hline $\mathrm{RX}=1+\mathrm{a} \mathrm{t}+\mathrm{b} \mathrm{t}^{2}$ & Wang e Sing \\
$\mathrm{RX}=\mathrm{a} \cdot \exp (-\mathrm{k} \cdot \mathrm{t})+(1-\mathrm{a}) \exp \left(-\mathrm{k}_{1} \cdot \mathrm{t}\right)$ & Verma \\
$\mathrm{RX}=\exp \left(\left(-\mathrm{a}-\left(\mathrm{a}^{2}+4 \cdot \mathrm{b} \cdot \mathrm{t}\right)^{0,5}\right) / 2 \cdot \mathrm{b}\right)$ & Thompson \\
$\mathrm{RX}=\exp \left(-\mathrm{k} \cdot \mathrm{t}^{\mathrm{n}}\right)$ & Page
\end{tabular}




$$
\begin{array}{ll}
\mathrm{RX}=\exp (-\mathrm{k} \cdot \mathrm{t}) & \text { Newton } \\
\mathrm{RX}=\mathrm{a} \cdot \exp \left(-\mathrm{k} \cdot \mathrm{t}^{\mathrm{n}}\right)+\mathrm{b} \cdot \mathrm{t} & \text { Midilli } \\
\mathrm{RX}=\mathrm{a} \cdot \exp (-\mathrm{k} \cdot \mathrm{t})+\mathrm{c} & \text { Logarítmico } \\
\mathrm{RX}=\mathrm{a} \cdot \exp (-\mathrm{k} \cdot \mathrm{t}) & \text { Henderson e Pabis } \\
\mathrm{RX}=\mathrm{a} \cdot \exp (-\mathrm{k} \cdot \mathrm{t})+\mathrm{b} \cdot \exp \left(-\mathrm{k}_{\mathrm{o}} \cdot \mathrm{t}\right)+\mathrm{c} \cdot \exp \left(-\mathrm{k}_{1} \cdot \mathrm{t}\right) & \text { Henderson e Pabis modificado } \\
\mathrm{RX}=\mathrm{a} \cdot \exp (-\mathrm{k} \cdot \mathrm{t})+(1-\mathrm{a}) \exp (-\mathrm{k} \cdot \mathrm{a} \cdot \mathrm{t}) & \text { Exponencial de Dois Termos } \\
\mathrm{RX}=\mathrm{a} \cdot \exp \left(-\mathrm{k}_{\mathrm{o}} \cdot \mathrm{t}\right)+\mathrm{b} \cdot \exp \left(-\mathrm{k}_{1} \cdot \mathrm{t}\right) & \text { Dois Termos } \\
\mathrm{RX}=\mathrm{a} \cdot \exp (-\mathrm{k} \cdot \mathrm{t})+(1-\mathrm{a}) \cdot \exp (-\mathrm{k} \cdot \mathrm{b} \cdot \mathrm{t}) & \text { Aproximação da Difusão }
\end{array}
$$

em que: t: tempo de secagem, $\mathrm{h} ; \mathrm{k}, \mathrm{k}_{\mathrm{o}}, \mathrm{k}_{1}$ : constantes de secagem, $\mathrm{h}^{-1} ; \mathrm{a}, \mathrm{b}, \mathrm{c}, \mathrm{n}$ : coeficientes dos modelos.

O ajuste dos modelos matemáticos aos dados experimentais de secagem foi realizado por meio de análise de regressão não linear, pelo método Gauss-Newton, por meio de um programa computacional estatístico, adotando-se como critério para as aproximações iniciais dos coeficientes dos modelos os valores relatados na literatura para a modelagem de outros produtos agrícolas. Para determinar o grau de ajuste para cada temperatura de secagem foi considerada a significância dos coeficientes de regressão pelo teste $t$, adotando o nível de 5\% de significância, a magnitude do coeficiente de determinação $\left(\mathrm{R}^{2}\right)$, os valores do erro médio relativo $(\mathrm{P})$, erro médio estimado $(\mathrm{SE})$ e o teste de Qui-quadrado $\left(\chi^{2}\right)$ ao nível de significância de $5 \%$.

O modelo matemático da difusão líquida com a solução analítica para a forma geométrica esférica, com aproximação de oito termos foi ajustado aos dados experimentais de secagem do crambe, considerando-se o raio equivalente inicial dos grãos, mantendo-o constante ao longo da secagem

Os erros médios relativo e estimado, bem como o teste de Qui-quadrado, foram calculados conforme as seguintes expressões:

$\mathrm{P}=\frac{100}{\mathrm{n}} \sum \frac{|\mathrm{Y}-\hat{\mathrm{Y}}|}{\mathrm{Y}}$

$\mathrm{SE}=\sqrt{\frac{\sum(\mathrm{Y}-\hat{\mathrm{Y}})^{2}}{\mathrm{GLR}}}$

$\chi^{2}=\sum \frac{(\mathrm{Y}-\hat{\mathrm{Y}})^{2}}{\mathrm{GLR}}$

em que: Y: valor de RX experimental; $\hat{\mathrm{Y}}$ : valor de $\mathrm{RX}$ estimado pelo modelo; n: número de observações experimentais; GLR: graus de liberdade do modelo (observações menos o número de parâmetros do modelo).

$\mathrm{RX}=\frac{\mathrm{X}^{*}-\mathrm{X}_{\mathrm{e}}^{*}}{\mathrm{X}_{\mathrm{i}}^{*}-\mathrm{X}_{\mathrm{e}}^{*}}=\frac{6}{\pi^{2}} \sum_{\mathrm{n}=1}^{\infty} \frac{1}{\mathrm{n}^{2}} \exp \left[\frac{\mathrm{n}^{2} \cdot \pi^{2} \cdot \mathrm{D} \cdot \mathrm{t}}{9} \cdot\left(\frac{3}{\mathrm{R}}\right)^{2}\right]$

em que: RX: razão de teor de água, adimensional; D: coeficiente de difusão efetivo, $\mathrm{m}^{2} \mathrm{~s}^{-1}$; $\mathrm{n}$ : número de termos; R: raio equivalente, $\mathrm{m}$ $(0,0037 \mathrm{~m})$; T: tempo, s.

O raio equivalente é definido como o raio de uma esfera com volume equivalente ao do grão. Obteve-se o volume de cada grão $\left(\mathrm{V}_{\mathrm{g}}\right)$ por meio da medição dos três eixos ortogonais (comprimento, largura e espessura), em trinta grãos, no início da secagem, com auxílio de um paquímetro digital com resolução de $0,01 \mathrm{~mm}$, de acordo com a expressão proposta por Mohsenin (1986): 
$\mathrm{V}_{\mathrm{g}}=\frac{\pi \cdot(\mathrm{A} \cdot \mathrm{B} \cdot \mathrm{C})}{6}$

em que: $\mathrm{V}_{\mathrm{g}}$ : volume do grão, $\mathrm{m}^{3}$; $\mathrm{A}$ : maior eixo do grão, $\mathrm{m}$; B: eixo médio do grão, $\mathrm{m}$; $\mathrm{C}$ : menor eixo do grão, $\mathrm{m}$.

A relação do aumento do coeficiente de difusão com a elevação da temperatura do ar de secagem foi descrita por meio da equação de Arrhenius.

$\mathrm{D}=\mathrm{D}_{\mathrm{o}} \cdot \exp \left(\frac{-\mathrm{E}_{\mathrm{a}}}{\mathrm{R} \cdot \mathrm{T}_{\mathrm{a}}}\right)$

em que: $\mathrm{D}_{\mathrm{o}}$ : fator pré-exponencial; $\mathrm{E}_{\mathrm{a}}$ : energia de ativação, $\mathrm{kJ}$ $\mathrm{mol}^{-1}$; R: constante universal dos gases, $8,134 \mathrm{~kJ} \mathrm{kmol}^{-1} \cdot \mathrm{K}^{-1} ; \mathrm{T}_{\mathrm{a}}$ : temperatura absoluta, $\mathrm{K}$.

Os coeficientes da expressão de Arrhenius foram obtidos linearizando a Equação $20 \mathrm{com}$ a aplicação do logaritmo da seguinte forma:

$\mathrm{LnD}=\mathrm{LnD}_{\mathrm{o}}-\frac{\mathrm{E}_{\mathrm{a}}}{\mathrm{R}} \cdot \frac{1}{\mathrm{~T}_{\mathrm{a}}}$
Os valores do coeficiente de difusão efetivo foram submetidos à análise de variância e ao teste de comparação de médias de Tukey a $5 \%$ de significância.

\section{RESULTADOS E DISCUSSÃO}

$\mathrm{Na}$ Figura 1 se apresentam as curvas de secagem em diversas condições de ar para o Crambe abyssinica. Verificou-se que os tempos de secagem das sementes de crambe considerando-se a redução do teor de água de 38,0 para 8,0 (\% b.s.), nas temperaturas de $35,45,60,75$ e $90{ }^{\circ} \mathrm{C}$ foram 13,75 ; 7,$75 ; 5 ; 3,75$ e $3,26 \mathrm{~h}$, respectivamente, e que, com o aumento da temperatura, ocorreu diminuição do tempo de secagem evidenciando a maior velocidade de retirada de água.

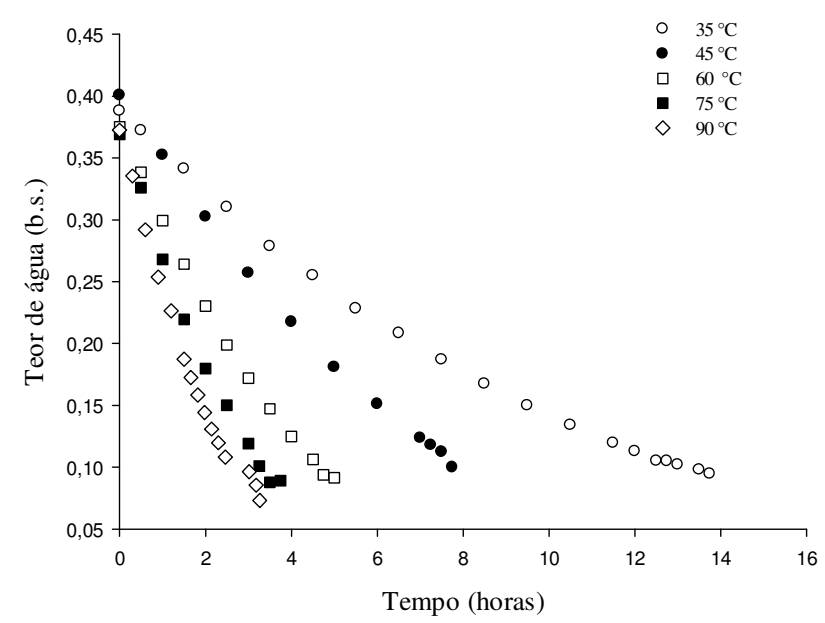

Figura 1. Curvas de secagem das sementes de Crambe abyssinica nas temperaturas de $35,45,60,75$ e $90{ }^{\circ} \mathrm{C}$.

Pode-se observar que a cinética de secagem do crambe apresenta as mesmas características da maioria dos grãos agrícolas, nabo (SOUSA et al., 2011), crambe (COSTA et al., 2011; COSTA et al., 2012b), ou seja, existe uma clara dependência da velocidade da secagem com a temperatura do ar. Segundo Peske et al. (2003), a utilização de temperaturas elevadas permite uma secagem mais rápida, porém pode provocar diferença de teor de água entre a periferia e o centro da semente, gerando um gradiente de pressão possível de causar danificação mecânica.

Na Tabela 2 se apresenta o resumo do ajuste dos doze modelos analisados por meio de regressão não linear aos dados experimentais de secagem de crambe em camada fina, considerando-se as diferentes temperaturas do ar de secagem.

Tabela 2. Erro médio estimado (SE), teste de $Q u i-q u a d r a d o ~\left(\chi^{2}, \times 10^{-3}\right)$, erro médio relativo $(\mathrm{P})$ e coeficiente de determinação $\left(\mathrm{R}^{2}\right)$, durante a secagem do crambe nas diversas condições de temperatura.

\begin{tabular}{|c|c|c|c|c|c|c|c|c|}
\hline \multirow{2}{*}{ Modelos } & SE & $\chi^{2}$ & $\mathrm{P}$ & $\mathrm{R}^{2}$ & SE & $\chi^{2}$ & $\mathrm{P}$ & $\mathrm{R}^{2}$ \\
\hline & \multicolumn{2}{|c|}{ (decimal) } & \multicolumn{2}{|c|}{$(\%)$} & \multicolumn{2}{|c|}{ (decimal) } & \multicolumn{2}{|c|}{$(\%)$} \\
\hline
\end{tabular}




\begin{tabular}{|c|c|c|c|c|c|c|c|c|}
\hline \multirow{3}{*}{ Wang e Singh } & \multirow{2}{*}{\multicolumn{4}{|c|}{$35^{\circ} \mathrm{C}$}} & \multirow{2}{*}{\multicolumn{4}{|c|}{$45^{\circ} \mathrm{C}$}} \\
\hline & & & & & & & & \\
\hline & 0,031 & 1,012 & 6,69 & 98,64 & 0,004 & 0,019 & 0,76 & 99,97 \\
\hline Verma & 0,129 & 16,795 & 26,92 & 78,94 & 0,183 & 3,354 & 41,87 & 62,74 \\
\hline Thompson & 0,026 & 0,719 & 4,71 & 99,03 & 0,024 & 0,578 & 4,62 & 99,27 \\
\hline Page & 0,033 & 1,114 & 6,29 & 98,47 & 0,003 & 0,015 & 0,80 & 99,98 \\
\hline Newton & 0,045 & 2,046 & 10,26 & 97,09 & 0,022 & 0,520 & 4,62 & 99,27 \\
\hline Midilli & 0,015 & 0,255 & 2,26 & 99,70 & 0,003 & 0,010 & 0,57 & 99,99 \\
\hline Logarítmico & 0,015 & 0,251 & 1,79 & 99,68 & 0,005 & 0,035 & 1,06 & 99,96 \\
\hline Henderson e Pabis & 0,044 & 2,000 & 9,64 & 97,32 & 0,019 & 0,383 & 3,64 & 99,52 \\
\hline H. e Pabis Modificado & 0,286 & 82,040 & 58,03 & 17,71 & 0,004 & 0,017 & 0,61 & 99,98 \\
\hline Ex. de Dois Termos & 0,029 & 0,861 & 5,85 & 98,84 & 0,024 & 0,578 & 4,62 & 99,27 \\
\hline Dois Termos & 0,016 & 0,261 & 1,73 & 99,69 & 0,022 & 0,493 & 3,64 & 99,52 \\
\hline \multirow[t]{2}{*}{ Ap. da Difusão } & 0,019 & 0,366 & 1,98 & 99,54 & 0,003 & 0,011 & 0,60 & 99,98 \\
\hline & \multicolumn{4}{|c|}{$60^{\circ} \mathrm{C}$} & \multicolumn{4}{|c|}{$75^{\circ} \mathrm{C}$} \\
\hline Wang e Singh & 0,004 & 0,023 & 0,82 & 99,97 & 0,013 & 0,181 & 2,41 & 99,79 \\
\hline Verma & 0,025 & 0,656 & 4,91 & 99,25 & 0,042 & 1,832 & 9,35 & 98,16 \\
\hline Thompson & 0,024 & 0,591 & 4,91 & 99,25 & 0,030 & 0,937 & 5,68 & 98,93 \\
\hline Page & 0,002 & 0,007 & 0,54 & 99,99 & 0,009 & 0,093 & 2,19 & 99,89 \\
\hline Newton & 0,023 & 0,537 & 4,91 & 99,25 & 0,028 & 0,832 & 5,68 & 98,93 \\
\hline Midilli & 0,002 & 0,006 & 0,43 & 99,99 & 0,011 & 0,121 & 2,16 & 99,89 \\
\hline Logarítmico & 0,007 & 0,058 & 1,49 & 99,93 & 0,014 & 0,222 & 2,61 & 99,77 \\
\hline Henderson e Pabis & 0,019 & 0,368 & 3,70 & 99,53 & 0,024 & 0,615 & 4,55 & 99,29 \\
\hline H. e Pabis Modificado & 0,082 & 6,856 & 15,49 & 94,81 & 0,035 & 1,229 & 4,55 & 99,29 \\
\hline Ex. de Dois Termos & 0,024 & 0,591 & 4,91 & 99,25 & 0,030 & 0,936 & 5,68 & 98,93 \\
\hline Dois Termos & 0,033 & 1,100 & 7,16 & 98,89 & 0,011 & 0,126 & 2,14 & 99,89 \\
\hline \multirow[t]{2}{*}{ Ap. da Difusão } & 0,001 & 0,003 & 0,39 & 99,99 & 0,016 & 0,259 & 2,60 & 99,74 \\
\hline & \multicolumn{4}{|c|}{$90^{\circ} \mathrm{C}$} & & & & \\
\hline Wang e Singh & 0,013 & 0,185 & 2,96 & 99,72 & & & & \\
\hline Verma & 0,022 & 0,499 & 3,95 & 99,32 & & & & \\
\hline Thompson & 0,021 & 0,461 & 3,95 & 99,32 & & & & \\
\hline Page & 0,014 & 0,201 & 3,03 & 99,70 & & & & \\
\hline Newton & 0,020 & 0,428 & 3,95 & 99,32 & & & & \\
\hline Midilli & 0,012 & 0,152 & 2,48 & 99,80 & & & & \\
\hline Logarítmico & 0,017 & 0,304 & 3,55 & 99,58 & & & & \\
\hline Henderson e Pabis & 0,017 & 0,324 & 3,54 & 99,52 & & & & \\
\hline H. e Pabis Modificado & 0,083 & 6,999 & 16,14 & 92,85 & & & & \\
\hline Ex. de Dois Termos & 0,021 & 0,461 & 3,95 & 99,32 & & & & \\
\hline Dois Termos & 0,019 & 0,383 & 3,56 & 99,52 & & & & \\
\hline Ap. da Difusão & 0,022 & 0,499 & 3,95 & 99,32 & & & & \\
\hline
\end{tabular}

Em todas as condições de secagem os valores do coeficiente de determinação $\left(R^{2}\right)$, foram superiores a $98 \%$ para os dois modelos (Midilli e Page), indicando, de acordo com Madamba et al. (1996), uma representação satisfatória do processo de secagem. O coeficiente de determinação não constitui sozinho, um bom critério para a seleção de modelos não lineares, para isto, os valores para o $\mathrm{SE}, \mathrm{P}$ e $\chi^{2}$ foram considerados na seleção do modelo.

Os modelos de Midilli e Page apresentaram os menores valores de SE, bem como a magnitude do $\mathrm{P}$ para esses modelos foram inferiores a $10 \%$. Os valores de $\mathrm{P}$ indicam $\mathrm{o}$ desvio entre valores experimentais em relação à curva estimada pelo modelo (KASHANI-NEJAD et al., 2007) e, segundo Mohapatra e Rao (2005), valores de P inferiores a $10 \%$ são recomendados para a escolha de modelos.

Verifica-se, ainda, que o modelo de Dois Termos, Thompson, Wang e Singh, Aproximação da Difusão e Logarítmico, também se ajustaram satisfatoriamente aos dados experimentais de secagem para todas as temperaturas e parâmetros analisados.

Dentre os modelos que tiveram os melhores parâmetros estatísticos (Midilli e Page), o modelo Page foi selecionado por ser uma equação mais 
simples e por ter todos os seus coeficientes significativos a $1 \%$ pelo teste $\mathrm{t}$, conforme apresentado na Tabela 2.

A Figura 2 apresenta o bom ajuste do modelo de Page na representação da razão de umidade em função do tempo para a secagem do crambe.

Corrêa et al. (2003) utilizando temperatura de $30,40,50$ e $60{ }^{\circ} \mathrm{C}$ em secagem de sementes de milho da cultivar BR 400 Superdoce (Zea mays L.) verificaram que o modelo de Page foi o que melhor se ajustou aos dados de secagem dessa cultivar. $\mathrm{O}$ estudo da cinética de secagem da casca da manga (Tommy atkins) em três temperaturas diferentes (50, 60 e $70{ }^{\circ} \mathrm{C}$ ) observada por Azoubel et al. (2008), mostraram que o aumento da temperatura favoreceu a transferência de massa, diminuindo o teor de água de equilíbrio dinâmico e o tempo de secagem. Estes pesquisadores verificaram que o modelo de Page representou adequadamente os dados experimentais da secagem.

A cinética de secagem do abacaxi em fatias foi influenciada pelas temperaturas de 50,60 e 70 ${ }^{\circ} \mathrm{C}$, e entre os modelos aplicados o de Page ajustouse melhor aos dados experimentais (HOFSKY et al., 2009). Para Corrêa et al. (2006), os modelos de Page e Verma foram os que melhor representaram o fenômeno de secagem dos frutos de café da cultivar Mundo Novo da espécie Coffea arabica e Resende et al. (2009) analisando clones de café, recomendaram o modelo de Page para representação da secagem deste materiais.

Corrêa et al. (2007) verificaram que os modelos Page, Midilli, Henderson e Pabis modificado, Dois termos e Aproximação da difusão, foram os que melhor representaram o fenômeno de secagem do feijão nas temperaturas de 35,45 e 55 ${ }^{\circ} \mathrm{C}$, tendo sido o modelo de Page selecionado para descrever a cinética de secagem do produto.

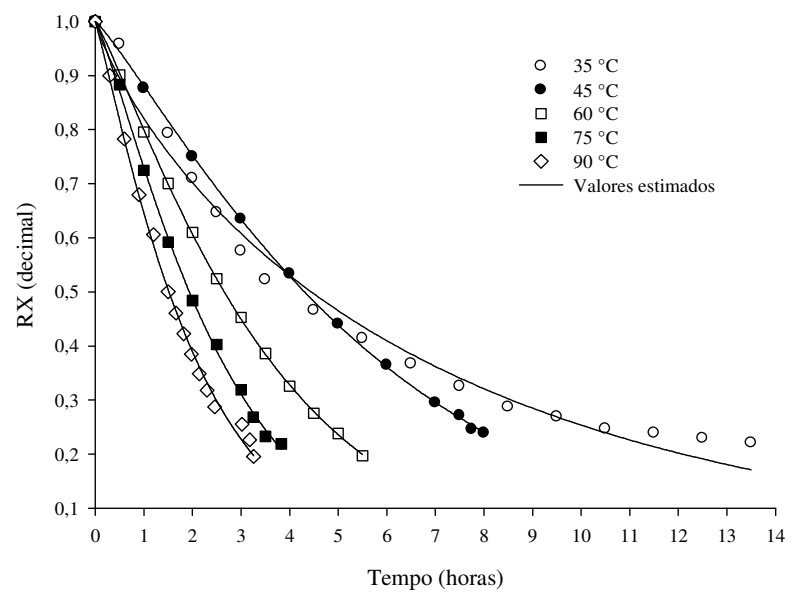

Figura 2. Valores da cinética de secagem dos frutos de Crambe abyssinica, experimentais e estimados pelo modelo de Page, para diversas condições de secagem.

Os coeficientes do modelo de Page ajustados aos dados experimentais da cinética de secagem dos frutos de crambe para diferentes condições de temperatura estão apresentados na Tabela 3. É possível observar que a constante de secagem " $k$ " variou quadraticamente em função da temperatura de secagem e o coeficiente " $n$ " oscilou com a variação da temperatura. Segundo Madamba et al. (1996) e Babalis e Belessiotis (2004), a constante de secagem $\mathrm{k}$ pode ser utilizada como aproximação para caracterizar o efeito da temperatura e está relacionada à difusividade efetiva no processo de secagem no período decrescente, sendo a difusão líquida que controla o processo.

Tabela 3. Coeficientes do modelo de Page ajustados as diversas temperaturas de secagens.

\begin{tabular}{ccc}
\hline \multirow{2}{*}{ Temperatura ${ }^{\circ} \mathrm{C}$} & \multicolumn{2}{c}{ Coeficientes } \\
\cline { 2 - 3 } & $\mathrm{k}$ & $\mathrm{N}$ \\
\hline 35 & $0,197470^{* *}$ & $0,841606^{* *}$ \\
45 & $0,127105^{* *}$ & $1,161341^{* *}$ \\
60 & $0,223585^{* *}$ & $1,158916^{* *}$ \\
75 & $0,314697^{* *}$ & $1,193730^{* *}$ \\
90 & $0,436337^{* *}$ & $1,111682^{* *}$ \\
Valores médios & $\mathrm{k}=0,3726-0,0098 \mathrm{~T}+0,0001 \mathrm{~T}^{2}$ & 1,0935 \\
\hline
\end{tabular}

** Significativo a $1 \%$ pelo teste $\mathrm{t}$. 
$\mathrm{Na}$ Figura 3 encontram-se os dados experimentais relacionados aos valores estimados pelo modelo de Page em função da temperatura de secagem. O modelo de Page mostrou-se apropriado para predição do fenômeno de secagem de frutos de crambe por apresentar os menores desvios e os melhores ajustes.

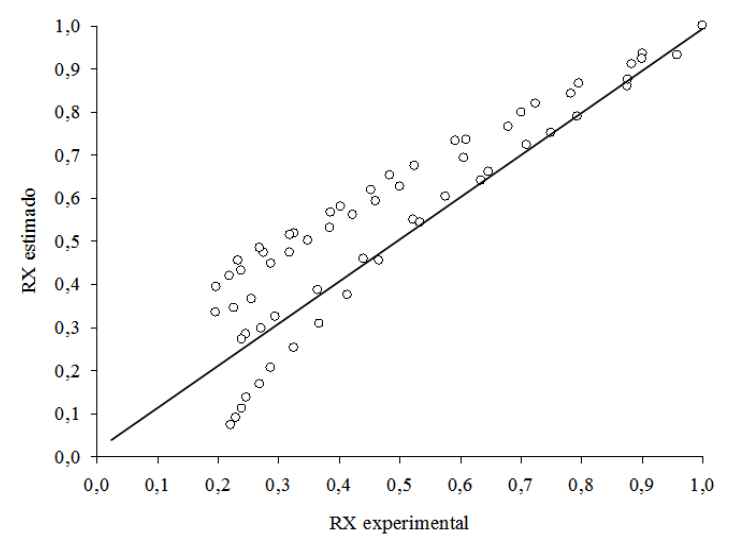

Figura 3. Valores experimentais relacionados aos valores estimados da razão de teor de água, obtidos pelo modelo de Page em função da temperatura de secagem do crambe.

A Figura 3 possibilita verificar o ajuste deste modelo na descrição da secagem do crambe. Observa-se uma correlação da razão de teor de água para os valores experimentais, entretanto, com a redução dos valores ocorrem maiores desvios entre os dados experimentais e os estimados pelo modelo; corroborando com os resultados obtidos por Costa et al. (2011), ao secarem frutos de crambe nas temperaturas de $30,40,50,60$ e $70{ }^{\circ} \mathrm{C}$, utilizando-se o modelo Wang e Singh.

Os valores da difusividade efetiva obtidos para as diferentes temperaturas de secagem dos frutos de Crambe abyssinica estão apresentados na Figura 4.

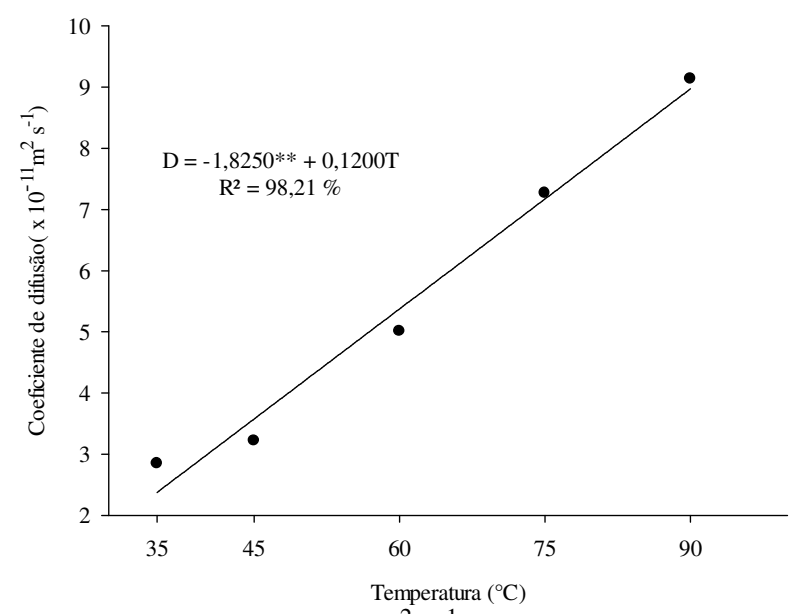

Figura 4. Valores médios do coeficiente de difusão $\left(\mathrm{m}^{2} \mathrm{~s}^{-1}\right)$ obtidos para a secagem do Crambe abyssinica nas temperaturas de $35,45,60,75$ e $90{ }^{\circ} \mathrm{C}$.

Os dados foram obtidos pela Equação 18 com aproximação de oito termos, a partir do qual os valores do coeficiente de difusão efetivo não apresentavam mais variação. A utilização de oito termos na equação está de acordo com Afonso Júnior e Corrêa (1999) que avaliaram os resultados de secagem de sementes de feijão ajustando o modelo da difusão para a forma geométrica esférica e verificaram adequada a aproximação da série para fornecer estimativas satisfatórias da taxa de secagem do produto.

Verifica-se que durante a secagem dos frutos de crambe o coeficiente de difusão efetivo aumenta $(\mathrm{P}<0,05)$, com a elevação da temperatura, apresentando valores de $2,84 \times 10^{-11} ; 3,22 \times 10^{-11}$; $5,00 \times 10^{-11} ; 7,27 \times 10^{-11}$ e $9,14 \times 10^{-11} \mathrm{~m}^{2} \mathrm{~s}^{-1}$, para as temperaturas de $35,45,60,75$ e $90{ }^{\circ} \mathrm{C}$, respectivamente. Os valores do coeficiente de difusão obtidos para os frutos de crambe estão 
coerentes com os relatados na literatura para secagem de produtos agrícolas, de acordo com Madamba et al. (1996). Costa et al. (2011), verificaram que, durante a secagem do crambe, o coeficiente de difusão efetivo também aumenta com a elevação da temperatura, apresentando valores de $0,88 \times 10^{-11}$ a $5,85 \times 10^{-11} \mathrm{~m}^{2} \mathrm{~s}^{-1}$, para as temperaturas entre 30 a $70{ }^{\circ} \mathrm{C}$. Faria et al. (2012), estudando a cinética de secagem do crambe observaram que para as temperaturas de $30 ; 40 ; 50$; 60 e $70{ }^{\circ} \mathrm{C}$, os valores do coeficiente de difusão efetivo apresentaram-se na ordem de $0,18 \times 10^{-10} \mathrm{a}$ $3,92 \times 10^{-10} \mathrm{~m}^{2} \mathrm{~s}^{-1}$. Durante a secagem do nabo forrageiro, os coeficientes de difusão apresentaram magnitudes entre $3,23 \times 10^{-11}$ e $10,43 \times 10^{-11} \mathrm{~m}^{2} \mathrm{~s}^{-1}$, para a faixa de temperatura de 30 a $70{ }^{\circ} \mathrm{C}$ (SOUSA et al., 2011).

Almeida et al. (2009), estudando a secagem de sementes de feijão adzuki, encontraram magnitudes de coeficientes de difusão efetivo entre $0,51 \times 10^{-10}$ e $2,23 \times 10^{-10} \mathrm{~m}^{2} \mathrm{~s}^{-1}$ para a faixa de temperatura de 30 a $70{ }^{\circ} \mathrm{C}$. O mesmo ocorreu para o feijão vermelho, que apresentou valores entre $2,1 \mathrm{x}$ $10^{-10}$ a $10,4 \times 10^{-10} \mathrm{~m}^{2} \mathrm{~s}^{-1}$ para temperaturas de secagem entre 25 e $55^{\circ} \mathrm{C}$ (RESENDE et al., 2007). Sacilik (2007) encontrou magnitudes de $8,53 \times 10^{-11}$ a $17,52 \times 10^{-11} \mathrm{~m}^{2} \mathrm{~s}^{-1}$ para o coeficiente de difusão das sementes de abóbora durante a secagem nas temperaturas de 40,50 e $60{ }^{\circ} \mathrm{C}$.

Os valores do coeficiente de difusão efetivo aumentaram linearmente com a elevação da temperatura corroborando aos resultados relatados por RESENDE et al. (2007), SOUSA et al. (2011) e COSTA et al. (2011), sua dependência com relação à temperatura do ar de secagem foi descrita pela equação de Arrhenius, Figura 5.

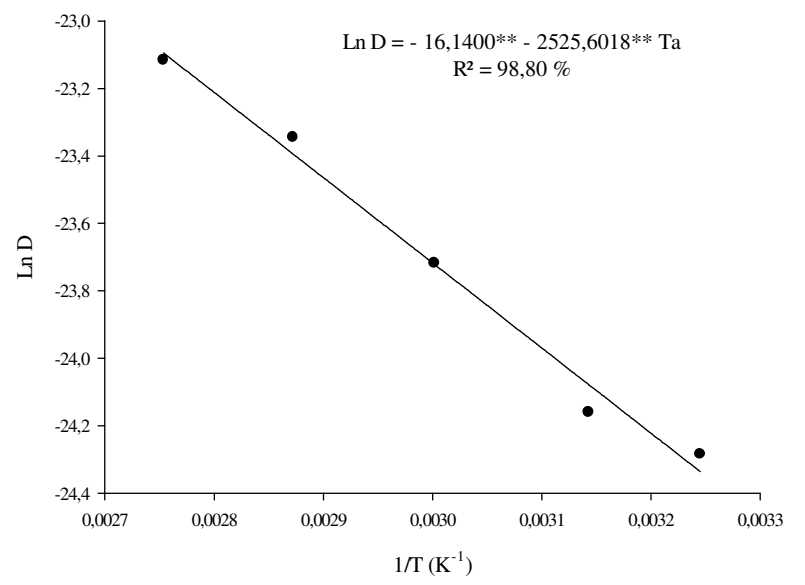

Figura 5. Representação de Arrhenius para o coeficiente de difusão, em função da temperatura do ar de secagem obtido durante a secagem do Crambe abyssinica.

A energia de ativação para a difusão líquida no processo de secagem do crambe foi de $20,998 \mathrm{~kJ}$ $\mathrm{mol}^{-1}$. A energia de ativação encontrada neste trabalho foi maior que a obtida por Faria et al. (2012), com o valor de $4,970 \mathrm{~kJ} \mathrm{~mol}^{-1}$ durante a secagem do crambe na faixa de temperatura de 30 a $70{ }^{\circ} \mathrm{C}$; e foi menor que as encontradas por Costa et al. (2011), sendo de $37,07 \mathrm{~kJ} \mathrm{~mol}^{-1}$ nas temperaturas de 30 a $70^{\circ} \mathrm{C}$ e nas sementes de nabo forrageiro sendo de $24,78 \mathrm{~kJ} \mathrm{~mol}^{-1}$ para a faixa de temperatura de 30 a $70{ }^{\circ} \mathrm{C}$ (SOUSA et al., 2011). Desta forma, pode-se dizer que a diferença da energia de ativação para a difusão líquida no processo de secagem para essas oleaginosas, possivelmente, ocorreu devido à diferença de teor de água inicial para cada produto e na faixa de temperatura utilizada.

Nos processos de secagem, quanto menor a energia de ativação maior também será a difusividade de água no produto. Segundo Kayacier e Singh (2004), a energia de ativação diminui com a elevação do teor de água inicial do produto durante o processo de secagem e de acordo com Kashaninejad et al. (2007), a energia de ativação é uma barreira que deve ser ultrapassada para que o processo de difusão possa ser desencadeado no produto. $\mathrm{O}$ ajuste linear obtido indica que houve uniformidade de variação da difusividade com a temperatura, de acordo com Reis et al. (2011), fato explicado em razão da vibração molecular de água. Segundo Goneli (2007), a variação do coeficiente de difusão efetivo se dá com a elevação da temperatura, o que aumenta o nível de vibração molecular das moléculas de água e contribui para uma difusão mais rápida. Pode-se dizer, portanto, que houve uma difusão maior na temperatura de 90 ${ }^{\circ} \mathrm{C}$. 


\section{CONCLUSÕES}

O comportamento das curvas de secagem dos frutos de crambe foi semelhante a maioria dos produtos agrícolas e o tempo de secagem foi de 13,$75 ; \quad 7,75 ; \quad 5,00 ; \quad 3,75$ e 3,26 horas, respectivamente, para as temperaturas de $35,45,60$, 75 e $90{ }^{\circ} \mathrm{C}$.

Analisando os resultados, conclui-se que, com base em parâmetros estatísticos, o modelo de Page foi selecionado para representação da secagem dos frutos de crambe.
O coeficiente de difusão efetivo aumenta com a elevação da temperatura, durante a secagem dos frutos de crambe e a dependência da difusividade em relação à temperatura foi descrita pela equação de Arrhenius, por meio da qual se obteve a energia de ativação de $20,998 \mathrm{~kJ} \mathrm{~mol}^{-1}$ para a difusão líquida no processo de secagem dos frutos de crambe.

\section{AGRADECIMENTOS}

Ao CNPq e a CAPES pelo apoio financeiro indispensável na execução do presente trabalho.

\begin{abstract}
The arm in this work was set several mathematical models to the process of drying the fruits of Crambe abyssinica in different air conditions and to determine the effective diffusion coefficient and obtain the activation energy. The work was performed at the Laboratory of Postharvest Products Vegetables of Instituto Federal de Educação, Ciência e Tecnologia Goiano - Câmpus Rio Verde, Goiás, Brasil (IF Goiano - Câmpus Rio Verde). The harvest of crambe (Crambe abyssinica Hochst) cultivar FMS Brilhante was performed manually with a water content of $38.0 \pm 1.0$ (\% d.b.) and dried until the content of $8.0 \pm 1.5$ (d.b.\% ). The drying procedure was conducted under different temperature controlled conditions of $35,45,60,75$ and $90{ }^{\circ} \mathrm{C}$ and relative humidity of $20.9,8.7,6.8,4.8$ and $2.3 \%$ respectively. Among the models that presented the best fit to the experimental data, Page was the best fit for the data in the different conditions of the drying air. The effective diffusion coefficient increased with increasing temperature with values of $2.84 \times 10^{-11}, 3.22 \mathrm{x}$ $10^{-11}, 5.00 \times 10^{-11}, 7.27 \times 10^{-11}$ and $9.14 \times 10^{-11} \mathrm{~m}^{2} \mathrm{~s}^{-1}$, at temperatures of $35,45,60,75$ and $90^{\circ} \mathrm{C}$, respectively, during the drying of crambe fruits. The relation between the effective diffusion coefficient and the drying temperature may be described by the Arrhenius equation, which has an activation energy for the liquid diffusion in drying of $20.998 \mathrm{~kJ} \mathrm{~mol}^{-1}$.
\end{abstract}

KEYWORDS: Crambe abyssinica. Air drying. Diffusion coefficient. Activation energy.

\title{
REFERÊNCIAS
}

ALMEIDA, D. P.; RESENDE, O.; COSTA, L. M.; MENDES, U. C.; SALES, J. F. Cinética de secagem do feijão adzuki (Vigna angularis). Global Science and Technology, Rio Verde, v. 2, n. 1, p. 72-83, 2009.

AFONSO JÚNIOR, P. C.; CORRÊA, P. C. Comparação de modelos matemáticos para descrição da cinética de secagem em camada fina de sementes de feijão. Revista Brasileira de Engenharia Agrícola e Ambiental, Campina Grande, v. 3, n. 3, p. 349-53, 1999

AZOUBEL, P. M.; EVANGELISTA, E. C. D.; OLIVEIRA, S. B.; SILVA, I. R. A.; ARAÚJO, A. J. B. Cinética de secagem da casca de manga (Tommy atkins). XVII Congresso Brasileiro de Engenharia Química. Recife - PE. de 14 a 17 de setembro de 2008.

BABALIS, S. J.; BELESSIOTIS, V. G. Influence of the drying conditions on the drying constants and moisture diffusivity during the thin-layer drying of figs. Journal of Food Engineering, Londres, v. 65, n. 3, p. 449-58, 2004. http://dx.doi.org/10.1016/j.jfoodeng.2004.02.005

BAEZ, O. "Crambe" a grande aposta das pesquisas em Mato Grosso do Sul. Jornal Pantanal news, 22 de agosto de 2007. Disponível em: <http://www.biodieselbr.com/noticias/em-foco/crambe-grande-apostapesquisasmato-grosso-sul.htm> Acesso em: 15 mar. 2011.

BRASIL, Ministério da Agricultura e Reforma Agrária. Secretaria Nacional de Defesa Agropecuária. Regras para análise de sementes. Brasília: Mapa/ACS. 2009. 395p. 
CORRÊA, P. C.; RESENDE, O.; MARTINAZO, A. P.; GONELI, A. L. D.; BOTELHO, F. M.; Modelagem matemática para a descrição do processo de secagem do feijão (Phaseolus vulgaris L.) em camadas delgadas. Engenharia Agrícola, Jaboticabal, v. 27, n. 2, p. 501-510, 2007.

CORRÊA, P. C.; RIBEIRO, D. M.; RESENDE, O.; BOTELHO, F. M. Determinação e modelagem das propriedades físicas e da contração volumétrica do trigo, durante a secagem. Revista Brasileira de Engenharia Agrícola e Ambiental, Campina Grande, v. 10, n. 3, p. 665-670, 2006

CORRÊA, P. C.; ARAÚJO, E. F.; AFONSO JÚNIOR, P. C. Determinação dos parâmetros de secagem em camada delgada de sementes de milho doce (zea mays 1.). Revista Brasileira de Milho e Sorgo, Sete Lagoas, v. 2, n. 2, p. 110-119, 2003.

COSTA, L. M.; RESENDE, O.; GONÇALVES, D. N.; OLIVEIRA, D. E. C. Equilíbrio Higroscópico dos Frutos de Crambe. $5^{\circ}$ Congresso da Rede Brasileira de Tecnologia de Biodiesel. $8^{\circ}$ Congresso Brasileiro de Plantas Oleaginosas, Óleos, Gorduras e Biodiesel. Salvador - Bahia, 16 a 19 de abril de 2012a.

COSTA, L. M.; RESENDE, O.; GONÇALVES, D. N.; SOUSA, K. A.; SALES, J. F.; DONADON, J. R. The influence of drying on the physiological quality of crambe fruits. Acta Scientiarum. Agronomy, Maringá, v. 34 , n. 2, p. 213-218, 2012b.

COSTA, L. M.; RESENDE, O.; GONÇALVES, D. N.; SOUSA, K. A. Coeficiente de difusão efetivo para a secagem de sementes de crambe (Crambe abyssinica). Revista Brasileira de Engenharia Agrícola e Ambiental, Campina Grande, v. 15, n. 10, p. 1089-1096, 2011.

FARIA, R. Q.; TEIXEIRA, I. R.; DEVILLA, I. A.; ASCHERI, D. P. R.; RESENDE, O. Cinética de secagem de sementes de crambe, Revista Brasileira de Engenharia Agrícola e Ambiental, Campina Grande, v. 16, n. 5, p. 573-583, 2012.

FUNDAÇÃO MATO GROSSO DO SUL. Tecnologia e Produção: Crambe 2010. Maracajú: FUNDAÇÃO MS, 2010.

GINER, S. A.; MASCHERONI, R. H. Diffusive drying kinetics in wheat, Part 2: applying the simplified analytical solution to experimental data. Postharvest Technology, Amsterdam, v. 81, n. 1, p. 85-97, 2002.

GONELI, A. L. D. Dinâmica da variação das propriedades físicomecânicas e da qualidade das sementes de mamona (Ricinus communis $\mathbf{L}$.) durante a secagem e o armazenamento sob condições controladas. Viçosa: UFV, 2007. 192p. Tese Doutorado.

HOFSKY, A. V.; GOMES, J. P.; BARROS NETO, A. L.; SILVA, F. L. H.; ALMEIDA, F. A. C. Cinética de secagem de abacaxi cv pérola em fatias. Revista Brasileira de Produtos Agroindustriais, Campina Grade, v. 11, n. 2, p. 123-128, 2009.

KASHANI-NEJAD, M. A.; MORTAZAVI, A.; SAFEKORDI A. G. Thinlayer drying characteristics and modeling of pistachio nuts. Journal of Food Engineering, Londres, v. 78, n. 1, p. 98-108, 2007.

http://dx.doi.org/10.1016/j.jfoodeng.2005.09.007

KAYACIER, A.; SINGH, R. K. Application of effetive diffusivity approach for the moisture content prediction of tortilla chips during baking. Lebensmittel-Wissenschaft und- Technologie, London, v. 37, n. 2, p. 275-281, 2004.

MACHADO, M. F.; BRASIL, A. N.; OLIVEIRA, L. S.; NUNES, D. L. Estudo Do Crambe (Crambe abyssinica) Como Fonte De Óleo Para Produção De Biodiesel. In: II Congresso da Rede Brasileira de Tecnologia de Biodiesel, Anais, Brasília, 2007. 
MADAMBA, P. S.; DRISCOLL, R. H.; BUCKLE, K. A. Thin-layer drying characteristics of garlic slices. Journal of Food Engineering, Londres, v. 29, n. 1, p. 75-97, 1996. http://dx.doi.org/10.1016/02608774(95)00062-3

MARTINAZZO, A. P.; CORRÊA, P. C.; MELO, E. C.; BARBOSA, F. F. Difusividade efetiva em folhas de Cymbopogon citratus (DC.) Stapf submetidas à secagem com diferentes comprimentos de corte e temperaturas do ar. Revista Brasileira de Plantas Medicinais, Botucatu, v. 9, p. 68-72, 2007.

MOSHENIN, N. N. Physical properties of plant and animal materials. New York: Gordon and Breach Publishers. 1986. 841p.

MÖLLER, M. Crambe como Opção para Rotação de Cultura. Disponível em: <www.portaldoagronegocio.com.br/conteudo.php?id=11396. Acesso em: 18 jan. 2011.

MOHAPATRA, D.; RAO, P. S. A thin layer drying model of parboiled wheat. Journal of Food Engineering, Londres, v. 66, n. 4, p. 513-518, 2005. http://dx.doi.org/10.1016/j.jfoodeng.2004.04.023

PESKE, S. T.; VILLELA, F. Secagem de sementes. In: Peske, S. T.; Rosenthal, M.; Rota, G. R. M. Sementes: Fundamentos científicos e tecnológicos. Pelotas: Editora e Gráfica Universitária (UFPel). 2003. p. 283-322.

REIS, R. C.; BARBOSA, L. S.; LIMA, M. L.; REIS, J. S.; DEVILLA, I. A.; ASCHERI, D. P. R. Modelagem matemática da secagem da pimenta Cumari do Pará. Revista Brasileira de Engenharia Agrícola e

Ambiental, Campina Grande, v. 15, n. 4, p. 347-353, 2011.

RESENDE, O.; RODRIGUES, S.; SIQUEIRA, V. C.; ARCANJO, R. V. Cinética da secagem de clones de café (Coffea canephora Pierre) em terreiro de chão batido. Acta Amazônica, Manaus, v. 40, p. 247-256, 2010. http://dx.doi.org/10.1590/S0044-59672010000200002

RESENDE, O.; ARCANJO, R. V.; SIQUEIRA, V. C.; RODRIGUES, S. Modelagem matemática para a secagem de clones de café (Coffea canephora Pierre) em terreiro de concreto. Acta Scientiarum, Maringá, v. 31, n. 2, p. 189-196, 2009.

RESENDE, O.; CORREAA, P. C.; GONELI, A. L. D.; BOTELHO, F. M.; RODRIGUES, S. Modelagem matemática do processo de secagem de duas variedades de feijão (Phaseolus vulgaris L.). Revista Brasileira de Produtos Agroindustriais, Campina Grande, v. 10, n. 1, p. 17-26, 2008.

RESENDE, O.; CORRÊA, P. C.; JAREN, C.; MOURE, A. J. Bean moisture diffusivity and drying kinetics: a comparison of the liquid diffusion model when taking into account and neglecting grain shrinkage. Spanish Journal of Agricultural Research, Madri, v. 05, n. 1, p. 51-58, 2007.

ROCHA, D. C.; SANTOS, B. M.; SCHEID, C. M.; CALÇADA, L. A. Determinação dos coeficientes de difusão e convectivo na Secagem de sementes de nasturtium officinale (agrião). In: Congresso Brasileiro de Engenharia Química em Iniciação Científica, 6, 2005, Campinas. Resumos... Campinas: 2005.

SACILIK, K. Effect of drying methods on thinlayer drying characteristics of hull-less seed pumpkin (Cucurbita pepo L.). Journal of Food Engineering, Londres, v. 79, n. 1, p. 23-30, 2007.

http://dx.doi.org/10.1016/j.jfoodeng.2006.01.023

SOUSA, K. A.; RESENDE, O.; CHAVES, T. H.; COSTA, L. M. Cinética de secagem do nabo forrageiro (Raphanus sativus L.). Revista Ciência Agronômica, Fortaleza, v. 42, n. 4, p. 883-892, 2011. http://dx.doi.org/10.1590/S1806-66902011000400009

VILELA, C. A. A.; ARTUR, P. O. Secagem do açafrão (Curcuma longa L.) em diferentes cortes geométricos. Ciência e Tecnologia de Alimentos, Campinas, v. 28, n. 2, p. 387-394, 2008. http://dx.doi.org/10.1590/S010120612008000200018 
VECHIATTO, C. D.; FERNANDES, F. C. S. Aplicação de nitrogênio em cobertura na cultura do crambe. Revista Cultivando o saber, Cascavel, v. 4, n. 2, p. 18-24, 2011. 